Published in final edited form as:

Nat Methods. ; 9(5): 428-429. doi:10.1038/nmeth.1988.

\title{
Enhanced photostability of cyanine fluorophores across the visible spectrum
}

\author{
Roger B Altman ${ }^{1,5}$, Qinsi Zheng ${ }^{1,2,5}$, Zhou Zhou ${ }^{1}$, Daniel S Terry ${ }^{1,3}$, J David Warren $^{4}$, and \\ Scott C Blanchard ${ }^{1,2,3,4}$ \\ Scott C Blanchard: scb2005@med.cornell.edu \\ ${ }^{1}$ Department of Physiology and Biophysics, Weill Medical College of Cornell University, New \\ York, New York, USA \\ ${ }^{2}$ Tri-Institutional Training Program in Chemical Biology, Weill Medical College of Cornell \\ University, New York, New York, USA \\ ${ }^{3}$ Tri-Institutional Training Program in Computational Biology and Medicine, Weill Medical College \\ of Cornell University, New York, New York, USA \\ ${ }^{4}$ Department of Biochemistry, Weill Medical College of Cornell University, New York, New York, \\ USA
}

\begin{abstract}
To the Editor: Our recent demonstration ${ }^{1}$ that proximally linked 'protective' agents can yield substantial improvements in the performance of the cyanine fluorophore $\mathrm{Cy} 5$ motivated us to develop more photostable fluorophores spanning the visible spectrum. Such fluorophores would enable a broad range of applications and experimental demands. Here, using strategies identical to those previously described ${ }^{1}$, we show that marked enhancements in performance can also be achieved for other widely used members of the cyanine class, Cy2, Cy3, Cy3.5, Cy5.5 and Cy7 (Fig. 1a) when coupled to individual cyclooctatetraene (COT), nitrobenzylalcohol (NBA) and Trolox molecules (Supplementary Methods).
\end{abstract}

\begin{abstract}
We quantified each fluorophore's performance (Fig. 1b) using single-molecule fluorescence imaging techniques ${ }^{1}$. This method gives access to a number of salient properties of individual fluorophores under continuous laser excitation including the apparent brightness of fluorescent states and the rates at which transient and permanent nonfluorescent states are entered ('blinking' and 'photobleaching', respectively). These measures can then be used to assess the average number of photons emitted by a fluorophore before entering a dark state (the duration of fluorescence $\left(t_{\mathrm{on}}\right) \times$ mean fluorescence intensity) and the effective signal-tonoise ratio (SNR) of fluorescence, a parameter that reports on the variance in a flourophore's photon emission rate (Fig. 1b). These data reveal that the performance of each cyanine-class fluorophore tested is enhanced when linked to single protective agents. Notably, these positive effects varied substantially for each fluorophore type. For instance, each of the COT-linked fluorophores showed dramatic enhancements in overall performance. For Cy2COT, we observed an $\sim 25$-fold increase in the number of photons emitted before a darkstate transition. For Cy3-COT and Cy3.5-COT, this parameter changed very little, but the SNR of fluorescence for both molecules increased approximately four- to fivefold. Cy5.5-
\end{abstract}

\footnotetext{
(C) 2012 Nature America, Inc. All rights reserved.

5 These authors contributed equally to this work.

Note: Supplementary information is available on the Nature Methods website.

COMPETING FINANCIAL INTERESTS

The authors declare no competing financial interests.
} 
COT and Cy7-COT showed 50- and 70-fold increases, respectively, in the number of photons emitted before entering a dark state. Consequently, the average Cy5.5-COT and Cy7-COT molecule could be continuously imaged for $\sim 3 \mathrm{~min}$ at an SNR $>7: 1$ (Fig. 1c). Consistent with our previous work ${ }^{1}$, we observed only modest improvements for most NBA-linked fluorophores. However, we observed an almost 70-fold enhancement of photon count for Cy7-NBA. Trolox, which shows the most favorable impact on the performance of the Cy5 fluorophore ${ }^{1}$, also had relatively modest beneficial effects for the other cyanineclass fluorophores.

These data provide compelling evidence that the strategy of proximally linking protective agents to the fluorogenic center to enhance its performance can be generalized. However, the high variability in a yield of benefits observed for the cyanine class suggests the need for a deeper understanding of how protective agents provide photostabilization. Plausible mechanisms for mediating enhancements in fluorophore performance include cycles of reduction and oxidation, triplet-triplet energy transfer and exciplex-type relaxation ${ }^{2}$. As suggested by Tinnefeld and Cordes $^{3}$, the enhancements in performance of Trolox-linked fluorophores may arise through favorable reductionoxidation cycles with the fluorogenic center. However, the results we obtained suggest that the benefits of such a mechanism may be restricted to the Cy5 and Cy5.5 fluorophores, which perhaps reflects an appropriate matching of their redox potentials. By contrast, we observed more substantial and general enhancements for each of the COT-linked cyanine fluorophores. Given the estimated triplet energy and redox potential of COT (unpublished data) and COT's propensity for triplettriplet energy transfer ${ }^{4}$, these findings suggest that a triplet-triplet energy transfer mechanism may be the most generally effective means for cyanine fluorophore photostabilization. Forward progress toward quantifying the relative weights and impacts of each pathway for distinct fluorophores will enable additional improvements in the activities of these known protective agents. Insights of this kind may also aid the discovery of new compounds and the implementation of new strategies to meet the specific demands of distinct fluorophore types. Ultimately, continued efforts on these fronts may yield strategies that can be generalized for tailoring fluorophore performance for a broad array of distinct experimental demands.

\section{Supplementary Material}

Refer to Web version on PubMed Central for supplementary material.

\section{Acknowledgments}

We thank the members of the Blanchard laboratory for their critical comments on the manuscript. This work was supported by US National Institutes of Health (3R01GM079238-06S1 to S.C.B.), the Tri-Institutional Training Program in Chemical Biology and the Tri-Institutional Training Program in Computational Biology and Medicine.

\section{References}

1. Altman RB, et al. Methods. 2012; 9:68-71.

2. Turro NJ, Ramamurthy V, Scaiano JC. Modern Molecular Photochemistry of Organic Molecules (University Science Books). 2010

3. Tinnefeld P, Cordes T. Nat. Methods. 2012; 9:426-427. [PubMed: 22543371]

4. Widengren J, Chmyrov A, Eggeling C, Lofdahl P, Seidel AM. J. Phys. Chem. A. 2007; 111:429_ 440. [PubMed: 17228891] 

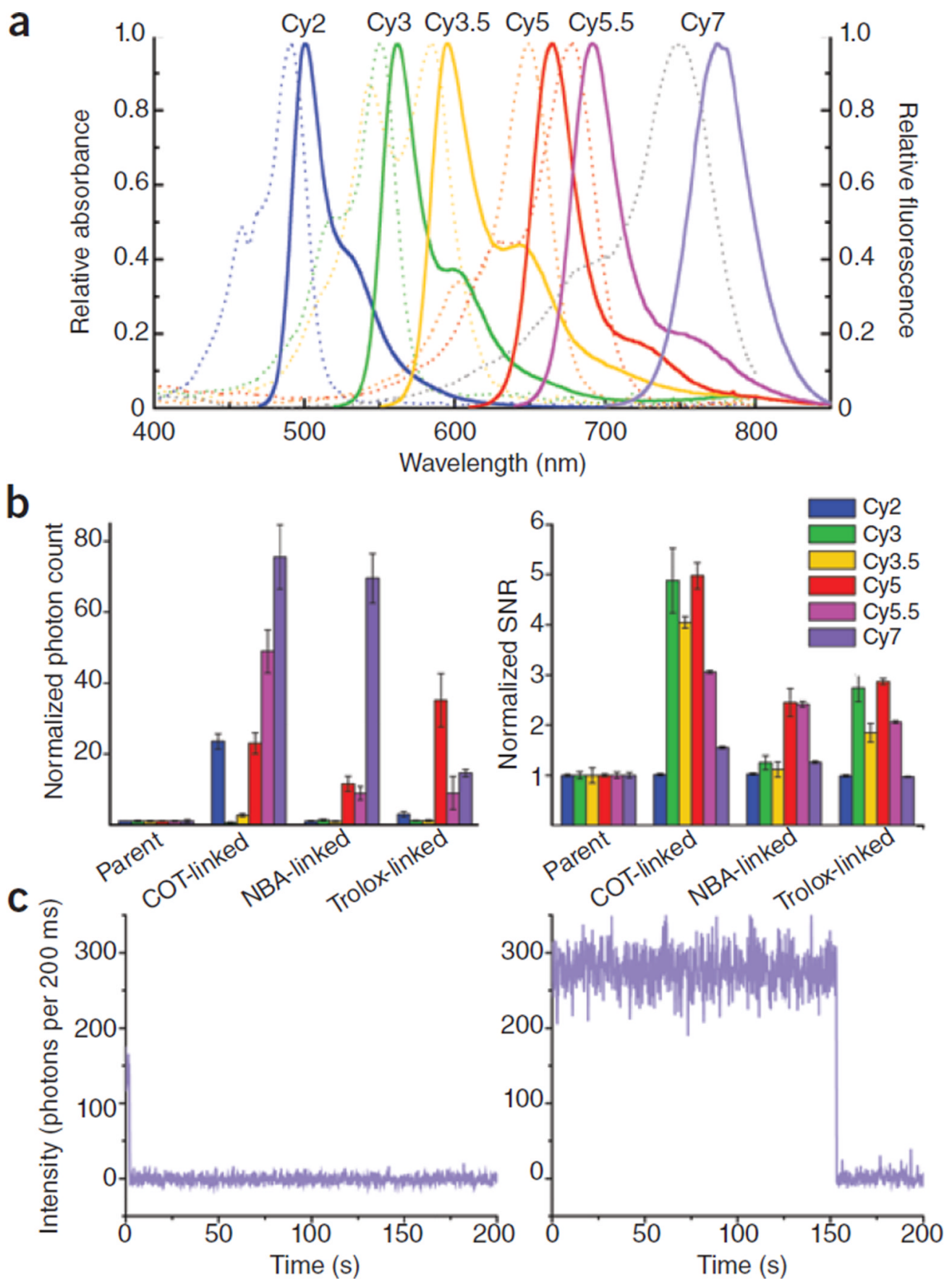

Figure 1.

Enhanced performance of distinct cyanine-class fluorophores through the direct conjugation to protective agents. (a) Absorbance (dotted lines) and fluorescence (solid lines) spectra of the cyanineclass fluorophores. (b) Average number of photons detected before the fluorophore entered dark states (left) and the average SNR of fluorescence, a measure of the variance in signal intensity over time (right), observed for the COT-, NBA- and Troloxlinked fluorophore derivatives of $\mathrm{Cy} 2, \mathrm{Cy} 3, \mathrm{Cy} 3.5, \mathrm{Cy} 5, \mathrm{Cy} 5.5$ and $\mathrm{Cy} 7$. All values were normalized to that of the corresponding parent compounds. Error bars, s.d. $(n=3$ experiments). (c) Representative Cy7 (left) and Cy7-COT (right) single-molecule 
fluorescence traces showing the average enhancement in performance ( $\sim 70$-fold) observed for Cy7-COT relative to Cy7. 